\title{
High confinement plasmas in the Madison Symmetric Torus reversed-field pinch $^{\text {a) }}$
}

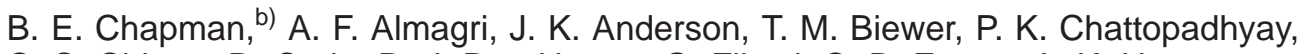 \\ C.-S. Chiang, D. Craig, D. J. Den Hartog, G. Fiksel, C. B. Forest, A. K. Hansen, \\ D. Holly, N. E. Lanier, R. O'Connell, S. C. Prager, J. C. Reardon, J. S. Sarff, \\ and M. D. Wyman \\ Department of Physics, University of Wisconsin-Madison, Madison, Wisconsin 53706 \\ D. L. Brower, W. X. Ding, Y. Jiang, and S. D. Terry \\ Department of Electrical Engineering, University of California-Los Angeles, Los Angeles, California 90095 \\ P. Franz, L. Marrelli, and P. Martin \\ Consorzio RFX, Associazione Euratom-Enea per la fusione, Corso Stati Uniti 4, 35127 Padova, Italy
}

(Received 1 November 2001; accepted 10 January 2002)

\begin{abstract}
Reduction of core-resonant $m=1$ magnetic fluctuations and improved confinement in the Madison Symmetric Torus [Dexter et al., Fusion Technol. 19, 131 (1991)] reversed-field pinch have been routinely achieved through control of the surface poloidal electric field, but it is now known that the achieved confinement has been limited in part by edge-resonant $m=0$ magnetic fluctuations. Now, through refined poloidal electric field control, plus control of the toroidal electric field, it is possible to reduce simultaneously the $m=0$ and $m=1$ fluctuations. This has allowed confinement of high-energy runaway electrons, possibly indicative of flux-surface restoration in the usually stochastic plasma core. The electron temperature profile steepens in the outer region of the plasma, and the central electron temperature increases substantially, reaching nearly $1.3 \mathrm{keV}$ at high toroidal plasma current $(500 \mathrm{kA})$. At low current $(200 \mathrm{kA})$, the total beta reaches $15 \%$ with an estimated energy confinement time of $10 \mathrm{~ms}$, a tenfold increase over the standard value which for the first time substantially exceeds the constant-beta confinement scaling that has characterized most reversed-field-pinch plasmas. (C) 2002 American Institute of Physics. [DOI: 10.1063/1.1456930]
\end{abstract}

\section{INTRODUCTION}

The reversed-field pinch is a toroidal magnetized plasma characterized in part by large radial magnetic shear and a toroidal magnetic field whose direction in the plasma edge is opposite that in the core. ${ }^{1}$ Magnetic islands are produced by multiple long-wavelength tearing modes with poloidal mode number $m=1$, resonant at various locations in the plasma core. The relatively small inter-island spacing and relatively large mode amplitudes lead to island overlap and stochastization of the magnetic field, allowing rapid radial energy transport.

The tearing modes are driven primarily by a gradient in the parallel current profile. With the goal of modifying this gradient in Madison Symmetric Torus (MST) ${ }^{2}$ reversedfield-pinch (RFP) plasmas, inductive auxiliary parallel current drive was applied at the plasma boundary. ${ }^{3-6}$ Comprised of a poloidal electric field induced by a transient change in the toroidal flux in the plasma, this technique resulted in a reduction of the $m=1$ fluctuations and an increase in the global energy confinement time to $5 \mathrm{~ms}$ from the MST standard $1 \mathrm{~ms}$.

Here, we report two advances in the understanding and control of magnetic fluctuations and energy transport. First,

\footnotetext{
${ }^{a)}$ Paper KI1 3, Bull. Am. Phys. Soc. 46, 173 (2001).

${ }^{b)}$ Invited speaker. Electronic mail: bchapman@ facstaff.wisc.edu
}

we now know that the previously achieved $m=1$ fluctuation reduction and confinement improvement were limited by bursts of edge-resonant $m=0$ magnetic fluctuations that were observed in conjunction with the auxiliary current drive. ${ }^{3-8}$ The $m=1$ fluctuations account for the bulk of energy transport in standard MST plasmas. ${ }^{9}$ Thus, the $m=0$ fluctuations have not previously been considered to play a significant role in transport (and their exact role is still not established). Second, we can now suppress these bursts, resulting in an additional improvement in energy confinement. Burst suppression is achieved through improved control of the surface parallel electric field, through manipulation of both the poloidal and toroidal electric fields. ${ }^{7,8}$ This has allowed a further reduction of the $m=1$ fluctuations, along with suppression of the $m=0$ bursts, for up to the duration of the auxiliary current drive.

With simultaneous control of core and edge fluctuations, high-energy runaway electrons are confined in the plasma core, possibly indicating at least a partial restoration of magnetic flux surfaces. The electron temperature profile steepens in the outer region of the plasma, and the central electron temperature increases substantially, reaching nearly $1.3 \mathrm{keV}$ at high toroidal plasma current $(500 \mathrm{kA})$. At low current (200 $\mathrm{kA})$ the total beta reaches about $15 \%$ with an estimated energy confinement time of $10 \mathrm{~ms}$, which is a tenfold increase over the MST standard confinement time and which substan- 


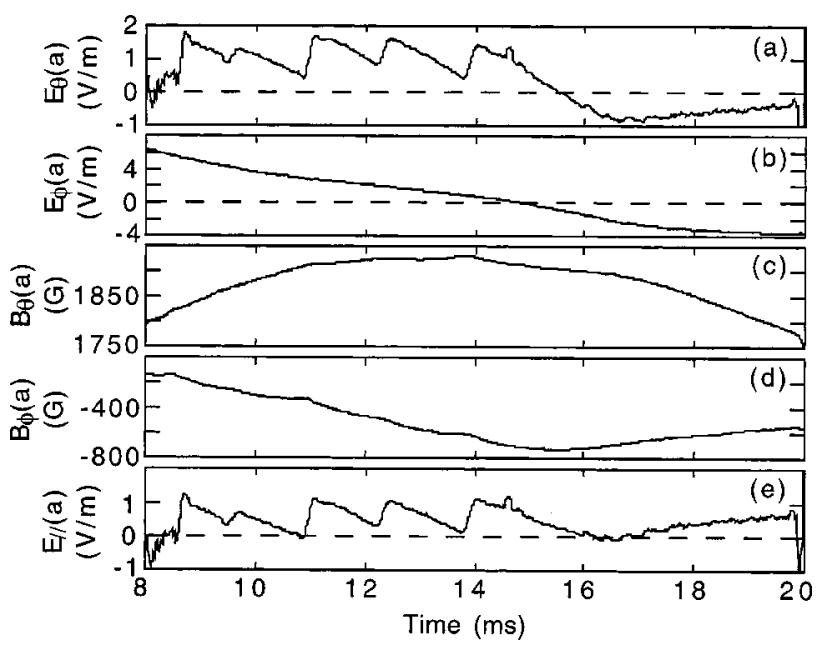

FIG. 1. Surface (a) poloidal and (b) toroidal electric fields, surface (c) poloidal, and (d) toroidal magnetic fields, and (e) surface parallel electric field in a $500 \mathrm{kA}$ plasma with auxiliary current drive beginning at about $8.5 \mathrm{~ms}$.

tially exceeds for the first time the confinement scaling that has characterized most RFP plasmas. ${ }^{8}$

In what follows, we discuss the application of and limitations on the inductive auxiliary current drive and describe the achieved fluctuation reduction. Following this are data illustrating the confinement of high-energy runaway electrons. Last, we describe the changes that occur in the temperature, density, energy confinement time, and beta.

\section{EXPERIMENTAL APPARATUS AND TECHNIQUE}

The ohmically heated MST RFP plasma has major and minor radii of 150 and $51 \mathrm{~cm}$, the latter determined by graphite limiters covering roughly $10 \%$ of the plasma-facing wall. The $5 \mathrm{~cm}$ thick conducting shell has a minor radius of $52 \mathrm{~cm}$ and also acts as the vacuum vessel and single-turn toroidal field winding. All of the plasmas described here were fueled with deuterium, but fluctuation reduction is also reliably achieved in hydrogen and helium.

We have established that sustained $m=0$ and $m=1$ fluctuation reduction requires $E_{\|} \geqslant 0$, where $E_{\|}=E_{\|}(a)=\mathbf{E} \cdot \mathbf{B} / B$ $=\left(E_{\theta} B_{\theta}+E_{\phi} B_{\phi}\right) / B$ is the surface parallel electric field, $E_{\theta}$ and $E_{\phi}$ are the surface poloidal and toroidal electric fields, $B_{\theta}$ and $B_{\phi}$ are the surface poloidal and toroidal magnetic fields, and $B$ is the total surface magnetic field. The wave forms of $E_{\theta}, E_{\phi}, B_{\theta}, B_{\phi}$, and $E_{\|}$from a discharge with auxiliary parallel current drive are shown in Fig. 1. From about $8.5-15 \mathrm{~ms}, E_{\|}$is maintained through the $E_{\theta} B_{\theta}$ term. Five consecutive triangular $E_{\theta}$ pulses are provided by the toroidal field circuit, which increases $\left|B_{\phi}(a)\right|$. During this time, $E_{\phi}$ is in the (normal) direction to sustain the toroidal plasma current in the core, making a negative contribution to $E_{\|}$in the edge. After $15 \mathrm{~ms}, E_{\phi}$ is reversed, further sustaining $E_{\|} \geqslant 0$ in the edge.

To better understand the role played by $E_{\phi}$, refer to Fig. 2(a), which contains the full magnetic field profiles estimated with a cylindrical equilibrium model for a plasma with a relatively large (negative) $B_{\phi}(a)$. A key location in the $B_{\phi}$ profile is the toroidal magnetic field reversal radius, $r_{\text {rev }}$,

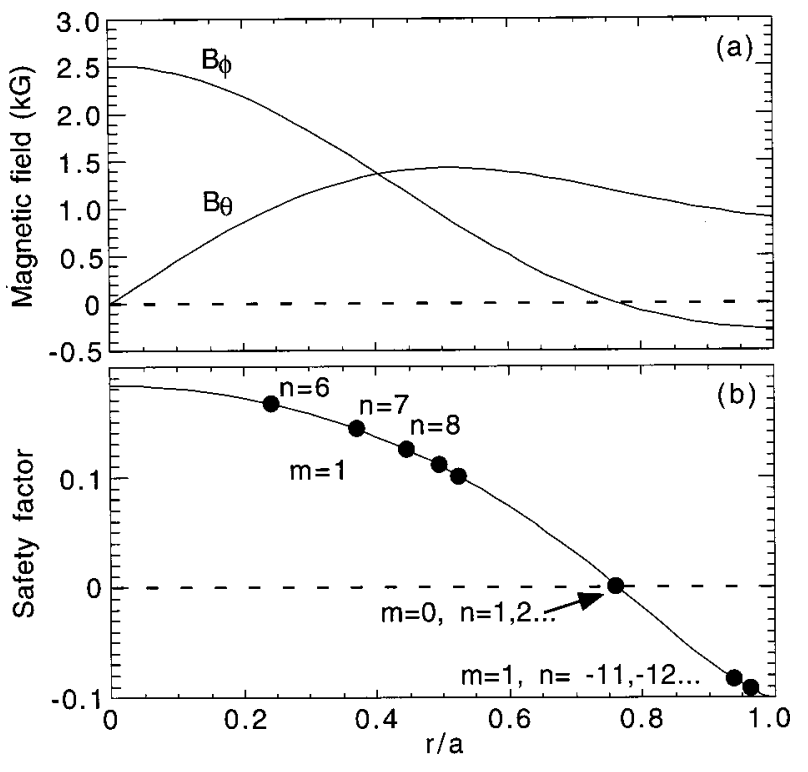

FIG. 2. Estimated profiles of (a) toroidal and poloidal magnetic fields, and (b) the safety factor for a $230 \mathrm{kA}$ plasma with strong toroidal magnetic field reversal. Locations of $m=1$ and $m=0$ resonant surfaces are indicated.

where $B_{\phi}(r)=0$. Across $r_{\text {rev }}$, the pitch of $B_{\phi}(r)$, and $\boldsymbol{B}(r)$, flips toroidally, such that the unidirectional toroidal electric field drives current in opposite directions, with respect to $\boldsymbol{B}$, in the regions $r<r_{\text {rev }}$ and $r>r_{\text {rev }}$. During the poloidal current drive phase in Fig. 1, $\left|E_{\theta} B_{\theta}\right|>\left|E_{\phi} B_{\phi}\right|$, allowing a net $E_{\|} \geqslant 0$. Near the end of the poloidal current drive, $B_{\phi}(a)$ is large and negative, allowing reversal of $E_{\phi}$ to contribute significantly to $E_{\|}$. Reversal of $E_{\phi}$ has little beneficial effect if $\left|B_{\phi}(a)\right|$ is small, as in standard RFP plasmas. In all cases, however, $E_{\phi}$ reversal brings about a forced termination of the discharge, as the central plasma current is no longer sustained. By properly spacing the $E_{\theta}$ pulses, and with the addition of $E_{\phi}$ reversal, we now satisfy the condition $E_{\|} \geqslant 0$ for over $10 \mathrm{~ms}$. It is allowed for $E_{\|}$to drop to zero for a brief time, as occurs twice in Fig. 1(e), while still maintaining reduced fluctuations.

Essentially independent of toroidal plasma current, the required $E_{\theta}$ peaks at about $1.5 \mathrm{~V} / \mathrm{m}$ (surface poloidal voltage of about $5 \mathrm{~V}$ ) in each pulse. The triangular shape of the $E_{\theta}$ pulses is not necessarily optimal, but we are as yet unable to produce different wave forms, such as a square pulse. Similarly independent of plasma current, $E_{\phi}$ reaches about -3 $\mathrm{V} / \mathrm{m}$ (surface toroidal voltage of about $-30 \mathrm{~V}$ ). Thus, we maintain $0 \leqslant E_{\|} \leqslant 1 \mathrm{~V} / \mathrm{m}$ throughout the auxiliary current drive. The increase in the reversed toroidal flux with poloidal current drive is reflected in rather extreme changes in two RFP equilibrium parameters, the reversal parameter, $F$ $\equiv B_{\phi}(a) /\left\langle B_{\phi}\right\rangle$, and the pinch parameter, $\Theta \equiv B_{\theta}(a) /\left\langle B_{\phi}\right\rangle$, where $\left\langle B_{\phi}\right\rangle$ is a cross-section average. In the (high-current) $500 \mathrm{kA}$ plasma in Fig. $1, F$ drops from -0.1 to -0.9 , and $\Theta$ increases from 1.7 to 2.4 . At low current (200 kA), $F$ reaches -2.1 , and $\Theta$ reaches 3.5.

Three operational requirements must be satisfied to achieve long periods of reduced fluctuations. Violation of these requirements usually results in continuous, not burst- 
like, increased $m=0$ fluctuations lasting the duration of the auxiliary current drive. One requirement is that $E_{\theta}$ not be too large, i.e., substantially in excess of the $1.5 \mathrm{~V} / \mathrm{m}$ peak described above. Another requirement is that the current drive not begin within about $1 \mathrm{~ms}$ of sawtooth crashes, which correspond to a brief, global increase in fluctuations and transport. ${ }^{10}$ To avoid crashes, we use a crash detection circuit that delays the start of the current drive until $>1 \mathrm{~ms}$ after a crash. Crashes occur less often after the toroidal plasma current has ramped up, so avoiding crashes during this time is simpler. However, large fluctuations are also triggered if the current drive is begun more than 1 to $2 \mathrm{~ms}$ after the peak of the plasma current. The reasons for these operational limitations are not yet established, but the latter two result in a few-ms-long time window for starting the auxiliary current drive.

There is also a soft density limit that increases weakly with plasma current. Just above the limit, discrete $m=0$ bursts occur with regularity. Still higher density results in continuous $m=0$ activity. In terms of the central lineaveraged density, $\left\langle n_{e}\right\rangle$, the limit ranges from $8 \times 10^{18} \mathrm{~m}^{-3}$ $-1.1 \times 10^{19} \mathrm{~m}^{-3}$ over a toroidal current range of $200-500$ $\mathrm{kA}$. Although we stop gas puffing before the current drive begins, the density still rises $\sim 50 \%$ with fluctuation reduction. We must, therefore, start the current drive with a density below the limit. Thorough wall conditioning (minimization of impurity influx) is also needed for fluctuation reduction, requiring a high-quality vacuum and pulsed discharge cleaning in helium, done overnight. Boronization ${ }^{11}$ has also been used but is not required. Perhaps also related to minimization of impurity influx, we must also control the radial magneticfield error that grows to large amplitude at the vertical cut in the conducting shell. ${ }^{12}$ At present, our error correction system is crude, allowing control of the error field for less than half the duration of the auxiliary current drive. We have found that minimization of the error at the start of the current drive is most essential, but this allows the error to grow large during fluctuation reduction. Substantial confinement improvement has been achieved in spite of this, but it is hoped that better error correction will result in further confinement improvement.

\section{FLUCTUATION REDUCTION}

The magnetic fluctuations affected by the auxiliary current drive are resonant at various locations across the plasma. In Fig. 2(b) is the safety factor $(q)$ profile calculated from the magnetic-field profiles in Fig. 2(a). In the core are $m=1$ fluctuations, the largest having toroidal mode numbers $n$ $=6-10$. All $m=0$ modes $(n=1,2,3 \ldots)$ are resonant at $r_{\text {rev }}$, where $q=m / n=0$. Because of the large $B_{\phi}(a)$ in these plasmas, $m=1$ modes of relatively low $n$ (but of a helicity opposite that of the core-resonant $m=1$ modes) also become resonant. With current drive at $200 \mathrm{kA}$, where $F$ reaches $-2.1, q(a)$ approaches -0.2 , bringing $m=1$ modes with $n$ $=-6,-7,-8 \ldots$ into resonance.

Differences between two $500 \mathrm{kA}$ plasmas with auxiliary current drive, but with and without $m=0$ activity, are illustrated in Fig. 3. In both plasmas, auxiliary current drive be-

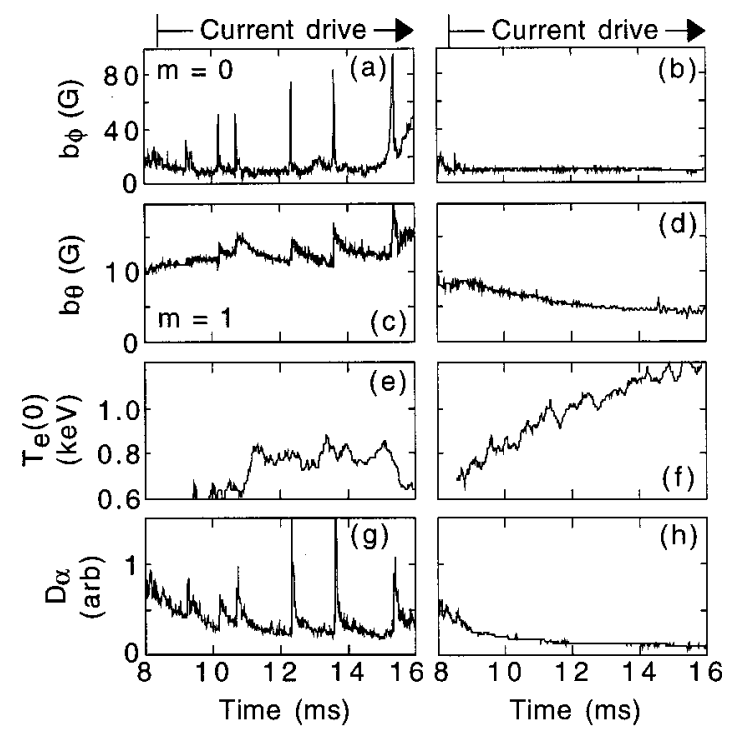

FIG. 3. In two $500 \mathrm{kA}$ plasmas with auxiliary current drive, (a) and (b) rms fluctuation in the toroidal magnetic field, including only $m=0, n=1-5$ modes, (c) and (d) rms fluctuation in the poloidal magnetic field, including only $m=1, n=6-15$ modes, (e) and (f) central electron temperature estimated from ratio of two x-ray intensities, calculated only when the x-ray intensity is nonzero, and $(\mathrm{g})$ and $(\mathrm{h})$ line radiation from neutral deuterium atoms. Oscillations in temperature due primarily to electronic noise in $\mathrm{x}$-ray signals.

gins at about $8.5 \mathrm{~ms}$. While there is essentially no $m=0$ activity in the plasma on the right, Fig. 3(b), the plasma on the left exhibits discrete $m=0$ bursts, Fig. 3(a). The bursts in this case occur due to the density slightly exceeding the soft limit, but the burst phenomenology is illustrative of that observed in previous current drive experiments, where the condition $E_{\|}(a) \geqslant 0$ was not satisfied continuously. Along with each $m=0$ burst is a corresponding increase in the $m=1$ modes. Such an increase is not seen with all $m=0$ bursts, but when it does occur, it usually follows the $m=0$ increase. One also observes that the $m=1$ modes fall off more slowly following each burst than do the $m=0$ modes. Sustained $m$ $=1$ reduction, such as that shown in Fig. 3(d), occurs only when $m=0$ bursts are suppressed. Thus, control of edgeresonant instability is essential to the improved-confinement plasmas described here.

To illustrate the effect of the differing fluctuation behavior on the plasma core, we plot the central electron temperature, $T_{e}(0)$, estimated from the ratio of soft-x-ray signals measured through beryllium filters of different thickness, Figs. 3(e) and 3(f). While there is no obvious response of $T_{e}(0)$ to the bursts, the temperature on the left is held to about $800 \mathrm{eV}$, while the temperature on the right, with sustained fluctuation reduction, increases steadily. The difference in $m=1$ fluctuation amplitudes probably plays a significant role in the temperature difference. To illustrate the effect of the $m=0$ bursts (and their absence) on the plasma edge, we compare the radiation from neutral deuterium in Figs. $3(\mathrm{~g})$ and $3(\mathrm{~h})$. The clear increases with each $m=0$ burst on the left are absent on the right.

The plasma on the right in Fig. 3 represents a very recent advance in fluctuation reduction. In general, there is usually 


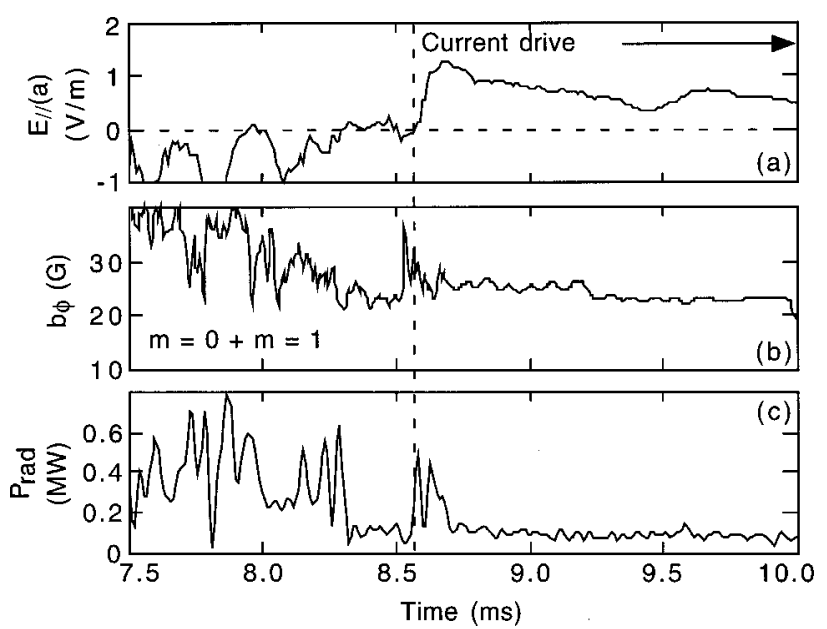

FIG. 4. From the plasma on the right in Fig. 3, (a) surface parallel electric field, (b) root-mean-square (rms) sum of $m=0$ and $m=1$ toroidal magnetic fluctuations with $n=1-15$, and (c) total radiated power (photons only).

a few-ms (transition) phase with enhanced $m=0$ activity following the start of auxiliary current drive. This delays the onset of fluctuation reduction, limiting the duration of the improved confinement and, e.g., the maximum achievable temperature and beta. This delay occurs when the current drive is begun during a standard-confinement MST plasma. The plasma on the right in Fig. 3 exhibits no such transition phase. The key difference here is that spontaneous improved confinement precedes the current drive. This is illustrated in Fig. 4, which contains data from the plasma on the right in Fig. 3. During the current drive, the toroidal magnetic fluctuations drop to around $25 \mathrm{G}$, and the radiated power drops to about $100 \mathrm{~kW}$ (such a low radiated power is achieved only during improved confinement). There is a similar reduction in these quantities for a brief period before the current drive begins, indicative of spontaneous improved confinement. Periods of spontaneous improved confinement, which can last up to $20 \mathrm{~ms}$, are also characterized by $m=0$ bursts and a reduction of magnetic fluctuations between bursts. ${ }^{71}{ }^{13-15}$ One burst occurs just as the current drive begins in Fig. 4. It is in $500 \mathrm{kA}$ plasmas like this that we have achieved a central electron temperature of about $1.3 \mathrm{keV}$, discussed more below. Such especially long periods of reduced fluctuations are not yet reliably achieved at lower current and do not contribute to the 200 and $400 \mathrm{kA}$ confinement data shown below.

Accompanying these global reductions in magnetic fluctuations is a global ten-fold reduction in electron density fluctuations. ${ }^{16}$ There is also a reduction of electrostatic (potential) fluctuations in the plasma edge. ${ }^{7,15}$ The relative contribution to improved energy confinement of magnetic and electrostatic fluctuation reduction remains to be determined.

\section{CONFINEMENT OF HIGH-ENERGY RUNAWAY ELECTRONS}

For the first time in an RFP, to our knowledge, the coreresonant magnetic fluctuations in the MST have been reduced to the point where high-energy runaway electrons can be confined. Evidence for the presence of these electrons lies in the emission of hard $x$ rays (HXR). In Fig. 5 is shown

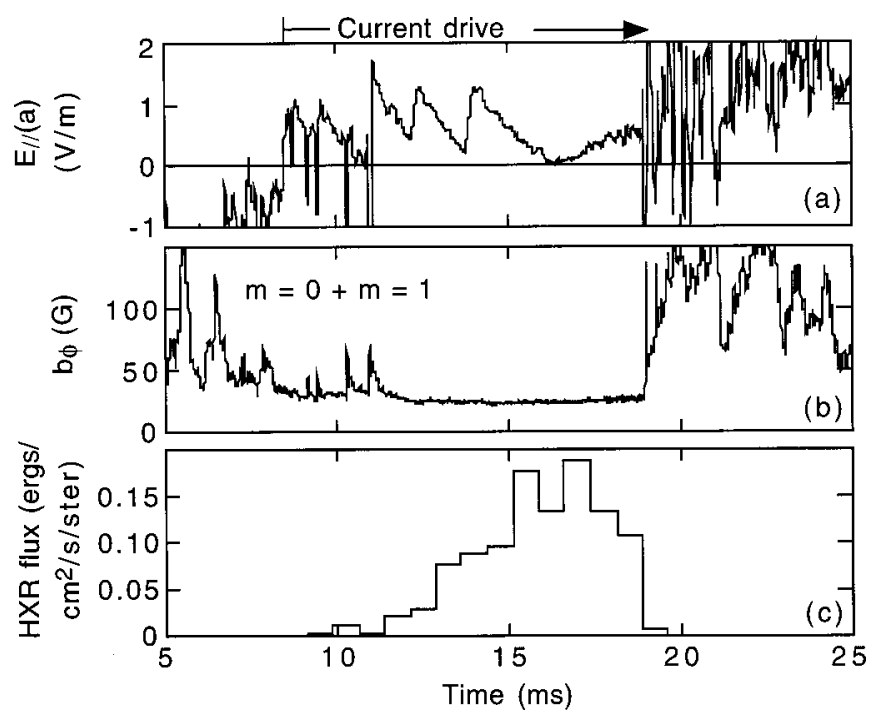

FIG. 5. From a $500 \mathrm{kA}$ plasma with auxiliary current drive (a) surface parallel electric field, (b) rms sum of $m=0$ and $m=1$ toroidal magnetic fluctuations with $n=1-15$, and (c) hard-x-ray flux.

HXR emission during a plasma with auxiliary current drive and a period of reduced magnetic fluctuations. At about 11 $\mathrm{ms}$, a period of sustained reduced magnetic fluctuations begins, and the $\mathrm{x}$-ray emission increases steadily. This emission is measured with a CdZnTe solid-state detector with a line of sight through the plasma core. The detector is sensitive to photons with energies from 10 to $250 \mathrm{keV}$. The photons in the MST are emitted as bremsstrahlung and reflect the energy of the runaway electrons from which they were emitted.

Nonzero HXR emission is only observed during periods of reduced fluctuations. In standard MST plasmas, the toroidal electric field is at least $1 \mathrm{~V} / \mathrm{m}$, sufficient to generate runaway electrons. However, the runaway electrons are apparently lost very rapidly, preventing them from accelerating to high energy. In the plasma in Fig. 5, the electrons reach an energy of over $70 \mathrm{keV}$, shown in the energy spectrum in Fig. 6. We have observed HXR emission up to $100 \mathrm{keV}$ in other plasmas. For electrons to accelerate to this energy requires well over 10000 toroidal transits around MST, a total distance greater than $100 \mathrm{~km}$. That such high-energy electrons can be confined may be an indication that there is at least a partial restoration of magnetic flux surfaces in the plasma core.

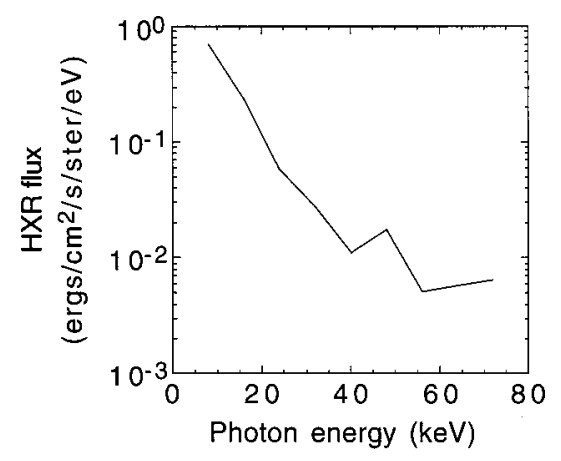

FIG. 6. Hard-x-ray energy spectrum from the plasma in Fig. 5. 


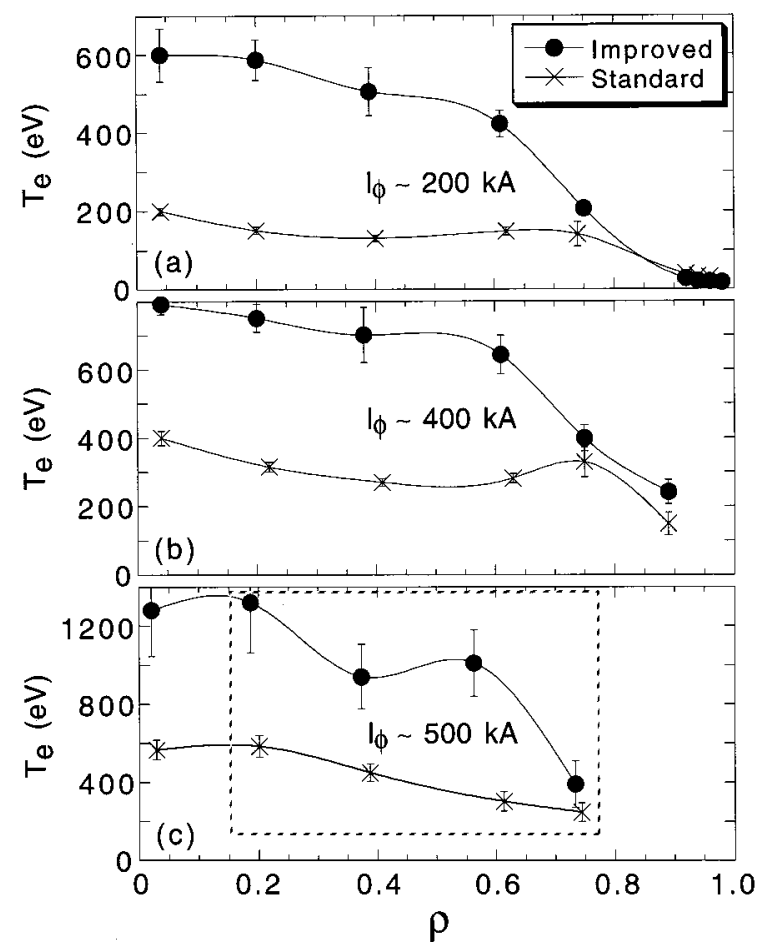

FIG. 7. Electron temperature profiles from standard- and improvedconfinement plasmas at three plasma currents with a central line-averaged density of (a) $\sim 8 \times 10^{18} \mathrm{~m}^{-3}$, (b) $1 \times 10^{19} \mathrm{~m}^{-3}$, and (c) $9 \times 10^{18} \mathrm{~m}^{-3}$. The central temperatures at $500 \mathrm{kA}$ are averages over more than ten shots, but the other $500 \mathrm{kA}$ data points (within the dashed box) are averages over less than five shots.

\section{CHANGES IN TEMPERATURE AND DENSITY}

With global fluctuation reduction, the electron temperature changes substantially, increasing in the core and decreasing in the edge. In contrast, the ion temperature changes little. The electron density profile flattens, sometimes to the point of becoming hollow, and like the edge temperature, the edge density also decreases. In this section, we compare plasmas with and without auxiliary current drive.

Electron temperature profiles from standard- and improved-confinement plasmas at three different plasma currents (and ohmic heating powers) are shown in Fig. 7. The data at $\rho<0.9$ were measured with a Thomson scattering diagnostic that provides $T_{e}$ at one location at one time in each plasma. The data at $\rho>0.9$, Fig. 7(a), were measured with a Langmuir probe. The data at 200 and $400 \mathrm{kA}$ are averages of measurements from many similar plasmas. At $500 \mathrm{kA}$, only the central-most data points are derived from many plasmas, but we include the other data points to show roughly how the profiles appear at this current. All three standard profiles were measured between sawtooth crashes at the peak of the plasma current when the plasma is hottest and densest. The three improved-confinement profiles were compiled at $18 \mathrm{~ms}$ in plasmas with long periods of reduced fluctuations. At all three currents, $T_{e}(0)$ increases substantially with a steepening of $T_{e}(r)$ in the region $\rho>0.6$. There is also a simultaneous decrease in the edge temperature, Fig. 7(a), measurable only at low current and shown in more detail below.

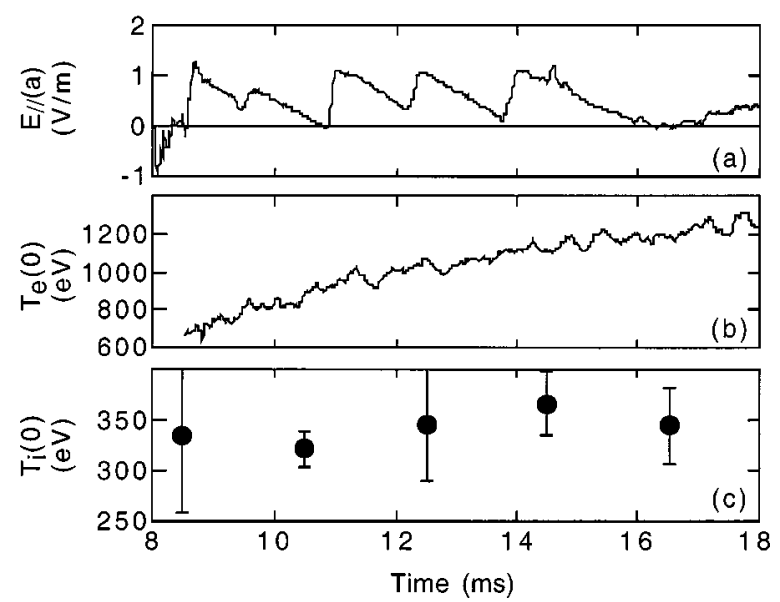

FIG. 8. (a) Surface parallel electric field and (b) central electron temperature from the $500 \mathrm{kA}$ plasma on the right in Fig. 3, and (c) central $\mathrm{C}^{+6}$ ion temperature compiled from many similar $500 \mathrm{kA}$ shots.

The central temperature reaches $1.28 \mathrm{keV}$ in the $500 \mathrm{kA}$ improved-confinement case. This is the first time that $T_{e}$ in a reversed-field-pinch plasma has exceeded $1 \mathrm{keV}$. The former largest shot-averaged RFP electron temperature, $860 \mathrm{eV}$, was achieved in standard plasmas in the TPE-1RM15 device. ${ }^{17}$ The MST temperature was achieved with a significantly lower (estimated) ohmic input power than in TPE-1RM15. Assuming a resistive loop voltage of $5 \mathrm{~V}$ in the MST plasmas, consistent with data for lower-current improvedconfinement plasmas in Table I (discussed below), the total electron heating power is $2.5 \mathrm{MW}$ at $500 \mathrm{kA}$. Using data from Ref. 17, and accounting for differences in plasma volume and electron density, one estimates that the heating power per electron in these MST plasmas is about 25 times lower than it was in TPE-1RM15, emphasizing the role of reduced electron energy loss in the MST, discussed more in the next section.

While $T_{e}$ evolves significantly with fluctuation reduction, the ion temperature, $T_{i}$, does not. The time evolution of $T_{i}(0)$, compiled point by point from many similar $500 \mathrm{kA}$ improved-confinement plasmas, is shown in Fig. 8. This is the temperature of $\mathrm{C}^{+6}$ ions, measured by charge exchange recombination spectroscopy. ${ }^{18}$ The temperature of the majority ions (deuterons), measured by Rutherford scattering, ${ }^{19}$ behaves similarly. Also plotted from a single plasma are $E_{\|}(a)$ and $T_{e}(0)$. Standard- and improved-confinement $T_{i}$ profiles are shown in Fig. 9. Similar to the $T_{e}$ profiles, the improved-confinement $T_{i}$ profile is flat in the core with a steep gradient near $\rho \sim 0.6$.

The electron density profile flattens considerably with fluctuation reduction. In Fig. 10 we compare density profiles from $500 \mathrm{kA}$ standard- and improved-confinement plasmas, measured with an 11-chord interferometer. These profiles are $1 \mathrm{~ms}$ time averages, and there is no gas puffing in either case. This flattening with fluctuation reduction is observed to a varying degree at all plasma currents.

While $n_{e}$ and $T_{e}$ increase in the plasma core with fluctuation reduction, they decrease in the edge. In Fig. 11 are edge profiles of $n_{e}$ and $T_{e}$ measured with a Langmuir probe 


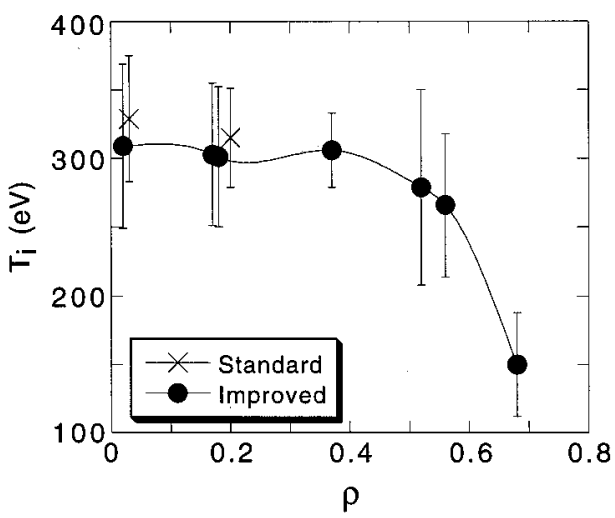

FIG. 9. $\mathrm{C}^{+6}$ ion temperature profiles in $500 \mathrm{kA}$ standard- and improvedconfinement plasmas.

in $200 \mathrm{kA}$ standard- and improved-confinement plasmas with the same $\left\langle n_{e}\right\rangle$ and no gas puffing. Both $n_{e}$ and $T_{e}$ decrease soon after the onset of fluctuation reduction and remain roughly constant thereafter.

\section{CONFINEMENT IMPROVEMENT}

The estimated energy confinement time, $\tau_{E} \equiv W_{\text {th }} /\left(P_{\text {oh }}\right.$ $\left.-\mathrm{d} W_{\mathrm{th}} / \mathrm{d} t\right)$, increases by up to a factor of ten with fluctuation reduction. Here, $W_{\text {th }} \equiv\left(\frac{3}{2}\right) \int\left(n_{e} T_{e}+n_{i} T_{i}\right) \mathrm{d} V$ is the volume-integrated plasma thermal energy, $n_{i}$ is the ion density, and $P_{\text {oh }}$ is the ohmic input power. The energy confinement time and its constituents are compared in Table I for standard- and improved-confinement plasmas at $\sim 200$ and $\sim 400 \mathrm{kA}$. We do not yet have sufficient data to calculate confinement parameters in $500 \mathrm{kA}$ plasmas. At $390 \mathrm{kA}, \tau_{E}$ is estimated to reach $8.8 \mathrm{~ms}$. At $210 \mathrm{kA}$ it reaches $10.1 \mathrm{~ms}$, the largest value yet achieved in the RFP and a tenfold increase over the MST standard $1 \mathrm{~ms}$, which is a time average including the time between and during sawtooth crashes. The corresponding global electron thermal diffusivity $\equiv a^{2} / 6 \tau_{E}$ drops from about 45 to $5 \mathrm{~m}^{2} / \mathrm{s}$. The estimated global electron particle confinement time, measured at $210 \mathrm{kA}$, increases eightfold, from 0.6 to $4.7 \mathrm{~ms}{ }^{16}$

Although the density increases with improved confinement, standard- and improved-confinement plasmas are com-

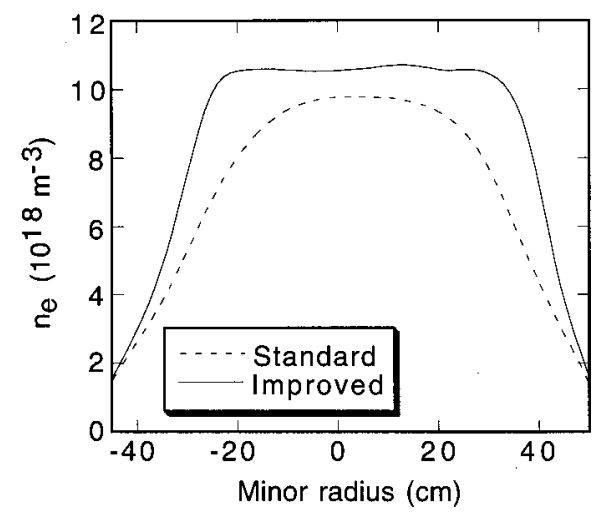

FIG. 10. Electron density profiles from standard- and improved-confinement $500 \mathrm{kA}$ plasmas. The profiles shown do not extend to the plasma boundary.

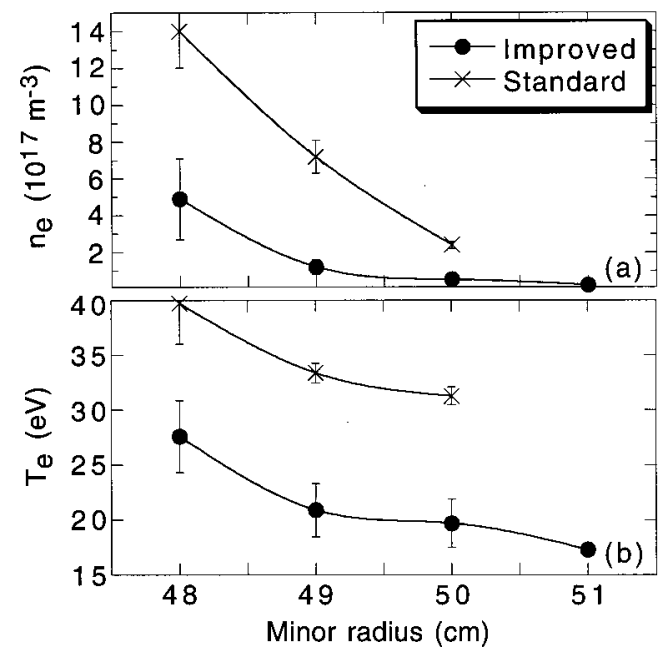

FIG. 11. Edge profiles of electron (a) density and (b) temperature from 200 kA standard- and improved-confinement plasmas with the same lineaveraged density. Plasma boundary at $51 \mathrm{~cm}$.

pared at similar densities by applying auxiliary current drive to plasmas with an initially reduced density. The central electron temperatures listed in Table I are extracted from the profiles in Fig. 7. The central ion temperature in the plasmas summarized in Table I was measured with Rutherford scattering. In the 210 and $430 \mathrm{kA}$ standard plasmas, $T_{i}(0)$ $=(0.75) T_{e}(0)$. In $210 \mathrm{kA}$ improved-confinement plasmas, $T_{i}(0)=(0.3) T_{e}(0)$, while at $390 \mathrm{kA}, T_{i}(0)=(0.5) T_{e}(0)$. Lacking $T_{i}$ profile measurements (partial profiles are available only in the recent $500 \mathrm{kA}$ plasmas), we assume the above ratios for the ion temperature profile, e.g., $T_{i}(r)$ $=(0.75) T_{e}(r)$ in standard plasmas. In these lower current plasmas, as in the $500 \mathrm{kA}$ plasmas, $T_{i}(0)$ changes little with fluctuation reduction. This may reflect an improvement in ion energy confinement, since at least one source of ion energy is expected to drop with improved confinement. In standard plasmas, the ions are heated via collisions with electrons and via an additional anomalous mechanism, the physics of which is not established but which is commonly linked to magnetic fluctuations. ${ }^{20}$ With fluctuation reduction, the anomalous heat input is expected to drop.

TABLE I. Parameters, defined in the text, from discharges with standard and improved confinement. Standard data measured between sawtooth crashes, except for $\tau_{E}$, where values between crashes and including crashes are shown. Improved-confinement $\left\langle n_{e}\right\rangle, T_{e}(0), \beta_{\text {tot }}$, and $\beta_{\theta}$ quoted at $18 \mathrm{~ms}$, when they peak, while $d W_{\text {th }} / d t$ quoted from 16 to $18 \mathrm{~ms}$, and $P_{\text {oh }}$ and $\tau_{E}$ quoted at $17 \mathrm{~ms}$, with an estimated uncertainty of $+/-2.5 \mathrm{~ms}$ for $\tau_{E}$.

\begin{tabular}{lcccc}
\hline \hline & Standard & Imp. & Standard & Imp. \\
\hline$I_{\phi}(k A)$ & 210 & 210 & 430 & 390 \\
$\left\langle n_{e}\right\rangle\left(10^{19} \mathrm{~m}^{-3}\right)$ & 0.8 & 0.7 & 1.0 & 1.0 \\
$T_{e}(0)(\mathrm{eV})$ & 200 & 600 & 400 & 792 \\
$d W_{\mathrm{th}} / d t(\mathrm{MW})$ & 0 & 0.47 & 0 & 0.55 \\
$P_{\mathrm{oh}}(\mathrm{MW})$ & 2.0 & 1.0 & 4.0 & 2.0 \\
$\tau_{E}(\mathrm{~ms})$ & $1.4 / 1.0$ & 10.1 & $1.6 / 1.0$ & 8.8 \\
$\beta_{\mathrm{tot}}(\%)$ & 9.0 & 15.4 & 4.8 & 10.7 \\
$\beta_{\theta}(\%)$ & 9.0 & 18.1 & 4.8 & 11.8 \\
\hline \hline
\end{tabular}


The ohmic input power drops by a factor of 2 with improved confinement (Table I). In standard plasmas, $P_{\text {oh }}$ is calculated through global power balance, where $P_{\text {oh }} \equiv P_{\text {in }}$ $-\mathrm{d} W_{m} / \mathrm{dt}, P_{\text {in }}$ is the total input power, and $W_{m}$ is the magnetic energy stored in the plasma. The total input power is the accurately measured Poynting flux at the plasma surface, and the relatively small $\mathrm{d} W_{m} / \mathrm{d} t$ is obtained from equilibrium modeling using measurements of the surface magnetic field. In the improved-confinement plasmas described here, $\mathrm{d} W_{m} / \mathrm{d} t \sim P_{\text {in }}$, and we lack the magnetic-field profile data required to accurately resolve the difference between these two terms. Thus, we estimate $P_{\text {oh }}$ for improved confinement through the volume integral $\int \eta J^{2} \mathrm{~d} V$, where $J$ is the current density, $\eta \propto Z / T_{e}^{3 / 2} /\left(1-f_{t}\right)$ is the plasma resistivity, $Z$ is calculated from $Z_{\text {eff }}$, the mean ionic charge, and $f_{t}$ is the trapped particle fraction. $J$ and $f_{t}$ are estimated at a single point in time with MSTFit, ${ }^{21}$ a toroidal equilibrium code whose calculations are constrained by measurements of, e.g., the pressure profile, the edge current profile, and the on-axis magnetic field (the latter measured with a motional-Starkeffect diagnostic ${ }^{18}$ ).

As an estimate of $Z_{\text {eff }}$ in improved-confinement plasmas, we adopt a value estimated in standard plasmas from a comparison of $P_{\text {oh }}$ calculated through power balance and $\int \eta J^{2} \mathrm{~d} V$ calculated with MSTFit. For example, we know from power balance that $P_{\mathrm{oh}}=4.0 \mathrm{MW}$ at $430 \mathrm{kA}$ in standard plasmas (Table I). MSTFit requires $Z_{\text {eff }}=2.0$, assumed spatially constant, to arrive at $\int \eta J^{2} \mathrm{~d} V=4.0 \mathrm{MW}$. The same comparison for the $210 \mathrm{kA}$ standard plasmas yields $Z_{\text {eff }}$ slightly lower than 2.0. This value is consistent with an upper-bound estimate of the central $Z_{\text {eff }}$ provided by the Rutherford-scattering diagnostic. For the improvedconfinement plasmas, we thus assume $Z_{\text {eff }}=2.0$ for both 210 and $390 \mathrm{kA}$.

The total beta, $\beta_{\text {tot }} \equiv 2 \mu_{0}\left[\int\left(n_{e} T_{e}+n_{i} T_{i}\right) \mathrm{d} V / \int \mathrm{d} V\right] / B^{2}(a)$ is shown in Table I. The poloidal beta, $\beta_{\theta}$, is similarly defined but with $B^{2}(a)$ replaced by $B_{\theta}^{2}(a)$. In standard plasmas, $B_{\theta}(a) \gg B_{\phi}(a)$, and $\beta_{\theta} \approx \beta_{\text {tot }}$, but in plasmas with auxiliary poloidal current drive, $B_{\phi}(a)$ becomes significant, and $\beta_{\theta}$ $>\beta_{\text {tot }}$. With improved confinement, both $\beta_{\text {tot }}$ and $\beta_{\theta}$ increase substantially. The improved-confinement betas at 210 $\mathrm{kA}$ are the largest yet achieved in the MST. We are as yet unable to quantify the energy stored in the runaway electrons. Without inclusion of this data, our quoted values of $\beta$, and possibly $\tau_{E}$, are underestimates.

The $\tau_{E}$ of $10 \mathrm{~ms}$ at $210 \mathrm{kA}$ significantly exceeds for the first time the scaling prediction of Connor and Taylor, where $\tau_{E}$ increases at constant beta and scales as $a^{2} I_{\phi}^{3} / N^{3 / 2}$, where $N=\pi a^{2}\left\langle n_{e}\right\rangle$ is the line density. This scaling is derived assuming that resistive fluid turbulence (g-modes) limits RFP energy confinement. ${ }^{22}$ In Fig. 12 are confinement data from different RFP devices. Most of these data were taken from Table I in Ref. 23, but the datum for TPE-1RM20 was taken from Ref. 24, and the data for the MST (a-e) are from the plasmas described in this paper and from the previously reported improved-confinement plasmas. ${ }^{5,6}$ The diagonal line is the best fit to the data listed in Ref. 23, $\tau_{E}$ $=10.2\left(a^{2} I_{\phi}^{3} / N^{3 / 2}\right)$. The previously achieved confinement times in the RFP have fallen on or somewhat below the

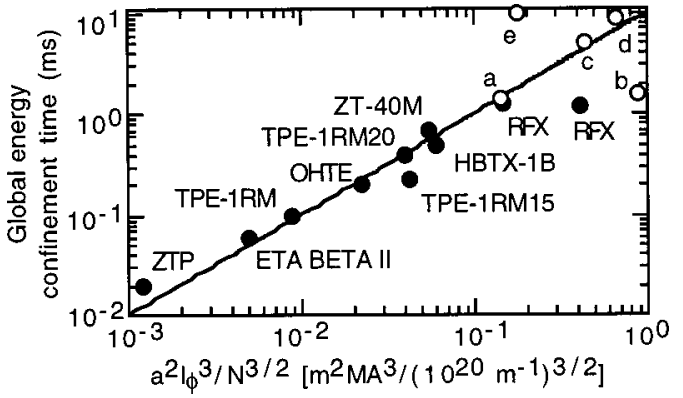

FIG. 12. Confinement results from various RFP devices and (a) MST 210 kA standard, (b) MST $430 \mathrm{kA}$ standard, (c) previous MST $340 \mathrm{kA}$ improved confinement, (d) present MST $390 \mathrm{kA}$ improved confinement, and (e) present MST $210 \mathrm{kA}$ improved confinement.

prediction of Connor-Taylor. In fact, $\tau_{E}$ in standard MST plasmas is essentially constant versus current and density. Hence, the achieved confinement increasingly diverges from the scaling as $I_{\phi}$ increases. The assumption of confinement limited by resistive, pressure-driven fluid turbulence has not been verified experimentally. The fact that improvedconfinement MST plasmas now exceed the scaling indicates that either the scaling is not generally valid or possibly that the assumed limiting turbulence has been reduced.

Another confinement scaling, $\tau_{E}=(1.9$ $\left.\times 10^{-5}\right) a^{1.4} \mu^{0.29} Z_{\text {eff }}{ }^{-0.42} I_{\phi}{ }^{0.34}$ was recently derived from nonlinear magnetohydrodynamic simulations of RFP plasmas without auxiliary current drive in which the coreresonant $m=1$ magnetic fluctuations limit confinement. ${ }^{25}$ Here, $\mu$ is the ion to proton mass ratio, and " $Z_{\text {eff }}$ " is meant to represent the $Z$ in Spitzer resistivity. The scaling is derived from a time average including sawtooth crashes, which were observed in the simulation. For MST plasmas, $\tau_{E}=(9.1$ $\left.\times 10^{-6}\right) Z_{\text {eff }}{ }^{-0.42} I_{\phi}{ }^{0.34} \approx 0.5 \mathrm{~ms}$ at $200 \mathrm{kA}$ and $0.6 \mathrm{~ms}$ at 400 $\mathrm{kA}$. These values are within a factor of 2 of the $1 \mathrm{~ms}$ experimental estimate for standard plasmas (Table I), and the scaling captures the essential independence of $\tau_{E}$ on $I_{\phi}$. As expected, the scaling prediction is significantly exceeded by the MST confinement times achieved with current drive and magnetic fluctuation reduction.

\section{SUMMARY AND CONCLUDING DISCUSSION}

With the realization that edge-resonant $m=0$ instability has previously limited confinement improvement, we have developed a technique to control this instability, leading to relatively long periods of reduced fluctuations. This fluctuation reduction allows confinement of high-energy runaway electrons, possibly indicating restoration of magnetic flux surfaces in the plasma core. The fluctuation reduction has also resulted in an increase in the core electron temperature with a steepening of the temperature gradient in the outer region of the plasma. At $500 \mathrm{kA}$, the central electron temperature reaches nearly $1.3 \mathrm{keV}$, representing the first time the temperature in an RFP has exceeded $1 \mathrm{keV}$. At $200 \mathrm{kA}$, the total beta reaches about $15 \%$ with an estimated energy confinement time of about $10 \mathrm{~ms}$, which is a tenfold increase 
over the MST standard confinement time and which substantially exceeds for the first time the confinement scaling that has characterized most RFP plasmas.

Auxiliary poloidal current drive has also been applied in the Reversed Field eXperiment (RFX) RFP, providing the first opportunity for inter-machine comparison. Applying five poloidal electric field pulses as in MST, RFX observed a reduction of $m=1$ fluctuations, an increase in the central electron temperature, and an improvement in energy confinement time, reaching about $2 \mathrm{~ms}^{26-28}$ Unlike the case in MST, however, the temperature profile became more peaked ( $\sim$ parabolic) and there was apparently no discrete $m=0$ activity. This may be due in part to the fact that RFX is limited to relatively high-density operation, with a typical $\left\langle n_{e}\right\rangle \sim 5$ $\times 10^{19} \mathrm{~m}^{-3}$, roughly five times the MST limit described above. Auxiliary poloidal current drive experiments have also just begun on TPE-RX. This device ${ }^{29}$ operates at densities similar to that of MST, so the phenomenology may be more similar to that in the MST.

Many questions remain as to the physics underlying these improved-confinement plasmas in the MST. Two interrelated issues are the source of the $m=0$ activity and the means by which this activity is curtailed. We speculate that the $m=0$ modes are driven linearly unstable by either the increased edge pressure or current ${ }^{30}$ gradients. If true, then the suppression of this activity by the current drive could be explained by either a modification of the edge current profile or, through ohmic heating, a modification of the pressure profile. Also yet to be established is the mechanism by which the $m=1$ fluctuations are reduced. The leading hypothesis is, of course, that the current profile is favorably modified by the current drive. Proof of this hypothesis requires detailed measurements of not only the current profile, but also the pressure, flow, and fluctuation profiles. This will be facilitated by the ongoing development of MST's profile diagnostics.

We believe that further improvements upon the present results are possible. For example, we believe that the finite duration of the auxiliary current drive may be limiting the maximum temperature, beta, and possibly the energy confinement time. Upgrades to inductive current drive may help to address this limitation. Work is also ongoing to develop radio-frequency current drive, using both lower-hybrid and electron-Bernstein waves, which has the potential for current drive in steady state.

\section{ACKNOWLEDGMENTS}

This work was supported by the U.S.D.O.E. The first author was supported in part by an appointment to the U.S.D.O.E.'s Fusion Energy Postdoctoral Research Program administered by the Oak Ridge Institute for Science and Education.
${ }^{1}$ H. A. B. Bodin and A. A. Newton, Nucl. Fusion 20, 1255 (1980).

${ }^{2}$ R. N. Dexter, D. W. Kerst, T. W. Lovell, S. C. Prager, and J. C. Sprott, Fusion Technol. 19, 131 (1991).

${ }^{3}$ J. S. Sarff, S. A. Hokin, H. Ji, S. C. Prager, and C. R. Sovinec, Phys. Rev. Lett. 72, 3670 (1994).

${ }^{4}$ J. S. Sarff, A. F. Almagri, M. Cekic et al., Phys. Plasmas 2, 2440 (1995).

${ }^{5}$ J. S. Sarff, N. E. Lanier, S. C. Prager, and M. R. Stoneking, Phys. Rev. Lett. 78, 62 (1997).

${ }^{6}$ M. R. Stoneking, N. E. Lanier, S. C. Prager, J. S. Sarff, and D. Sinitsyn, Phys. Plasmas 4, 1632 (1997).

${ }^{7}$ B. E. Chapman, Ph.D. thesis, University of Wisconsin-Madison, Madison, 1997.

${ }^{8}$ B. E. Chapman, J. K. Anderson, T. M. Biewer et al., Phys. Rev. Lett. 87, 205001 (2001).

${ }^{9}$ G. Fiksel, S. C. Prager, W. Shen, and M. Stoneking, Phys. Rev. Lett. 72, 1028 (1994).

${ }^{10}$ S. Hokin, A. Almagri, S. Assadi et al., Phys. Fluids B 3, 2241 (1991).

${ }^{11}$ D. J. Den Hartog, M. Cekic, G. Fiksel, S. A. Hokin, R. D. Kendrick, S. C. Prager, and M. R. Stoneking, J. Nucl. Mater. 200, 177 (1993); D. J. Den Hartog and R. D. Kendrick, ibid. 220-222, 631 (1995).

${ }^{12}$ A. F. Almagri, S. Assadi, S. C. Prager, J. S. Sarff, and D. W. Kerst, Phys. Fluids B 4, 4080 (1992).

${ }^{13}$ B. E. Chapman, A. F. Almagri, M. Cekic, D. J. Den Hartog, S. C. Prager, and J. S. Sarff, Phys. Plasmas 3, 709 (1996).

${ }^{14}$ B. E. Chapman, C.-S. Chiang, S. C. Prager, J. S. Sarff, and M. R. Stoneking, Phys. Rev. Lett. 80, 2137 (1998).

${ }^{15}$ B. E. Chapman, A. F. Almagri, J. K. Anderson et al., Phys. Plasmas 5, 1848 (1998).

${ }^{16}$ N. E. Lanier, D. Craig, J. K. Anderson, T. M. Biewer, B. E. Chapman, D. J. Den Hartog, C. B. Forest, S. C. Prager, D. L. Brower, and Y. Jiang, Phys. Rev. Lett. 85, 2120 (2000); Phys. Plasmas 8, 3402 (2001).

${ }^{17}$ Y. Hirano, Y. Yagi, T. Shimada, K. Hattori, Y. Maejima, I. Hirota, Y. Kondoh, K. Saito, and S. Shiina, Proceedings of the 13th International Conference on Plasma Physics and Controlled Nuclear Fusion Research, Washington DC, 1990 (International Atomic Energy Agency, Vienna, 1991), Vol. 2, p. 717.

${ }^{18}$ D. Craig, D. J. Den Hartog, G. Fiksel, V. I. Davydenko, and A. A. Ivanov, Rev. Sci. Instrum. 72, 1008 (2001).

${ }^{19}$ J. C. Reardon, G. Fiksel, C. B. Forest, A. F. Abdrashitov, V. I. Davydenko, A. A. Ivanov, S. A. Korepanov, S. V. Murachtin, and G. I. Shulzhenko, Rev. Sci. Instrum. 72, 598 (2001).

${ }^{20}$ E. Scime, S. Hokin, N. Mattor, and C. Watts, Phys. Rev. Lett. 68, 2165 (1992).

${ }^{21}$ J. K. Anderson, Ph.D. thesis, University of Wisconsin-Madison, Madison, 2001.

${ }^{22}$ J. W. Connor and J. B. Taylor, Phys. Fluids 27, 2676 (1984).

${ }^{23}$ K. A. Werley, J. N. DiMarco, R. A. Krakowski, and C. G. Bathke, Nucl. Fusion 36, 629 (1996).

${ }^{24}$ Y. Hirano, Y. Maejima, T. Shimada, I. Hirota, and Y. Yagi, Nucl. Fusion 36, 721 (1996).

${ }^{25}$ J. Scheffel and D. D. Schnack, Phys. Rev. Lett. 85, 322 (2000); Nucl. Fusion 40, 1885 (2000).

${ }^{26}$ R. Bartiromo, P. Martin, S. Martini, T. Bolzonella, A. Canton, P. Innocente, L. Marrelli, A. Murari, and R. Pasqualotto, Phys. Rev. Lett. 82, 1462 (1999).

${ }^{27}$ R. Bartiromo, V. Antoni, T. Bolzonella, A. Buffa, L. Marrelli, P. Martin, E. Martines, S. Martini, and R. Pasqualotto, Phys. Plasmas 6, 1830 (1999).

${ }^{28}$ S. Martini, D. Terranova, P. Innocente, and T. Bolzonella, Plasma Phys. Controlled Fusion 41, A315 (1999).

${ }^{29}$ Y. Yagi, Nucl. Fusion 40, 1933 (2000).

${ }^{30}$ B. E. Chapman, T. M. Biewer, P. K. Chattopadhyay et al., Phys. Plasmas 7, 3491 (2000). 